\title{
Efficiency of C-reactive protein in prognosis evaluation of prostate cancer: a systematic review and meta-analysis
}

\author{
Jianhui Du ${ }^{1 \#}$, Jianhua Lan ${ }^{1 \#}$, Jingjing Xiong ${ }^{2}$, Hai Yang ${ }^{1}$, Xiaohan Xu ${ }^{1}$, Chaolai Tang ${ }^{1}$, Guohua Huang ${ }^{1}$, \\ Qiao Ying ${ }^{1}$, Jian $\mathrm{Mu}^{1}$, Qiyi $\mathrm{Hu}^{3}$ \\ ${ }^{1}$ Department of Urology, People's Hospital of Guang'an City, Guang'an, China; ${ }^{2}$ Department of Urology, the First Affiliated Hospital of Chongqing \\ Medical University, Chongqing, China; ${ }^{3}$ Department of Urology, Affiliated Longhua People's Hospital, Southern Medical University (Longhua \\ People's Hospital), Shenzhen, China \\ Contributions: (I) Conception and design: Q Hu; (II) Administrative support: Q Hu; (III) Provision of study materials or patients: H Yang, Q Ying; \\ (IV) Collection and assembly of data: G Huang; (V) Data analysis and interpretation: J Du, J Lan; (VI) Manuscript writing: All authors; (VII) Final \\ approval of manuscript: All authors. \\ "These authors contributed equally to this work. \\ Correspondence to: Qiyi Hu. Department of Urology, Affiliated Longhua People's Hospital, Southern Medical University (Longhua People's Hospital), \\ 38 Jinglongjianshe Road, Longhua, Shenzhen 518109, China. Email: szhuqiyi@163.com.
}

\begin{abstract}
Background: Tumor markers can be diagnostically suggestive and are sometimes even related to prognostic prediction certain diseases. Multiple studies have shown the effectiveness in the prediction of prostate cancer $(\mathrm{PCa})$ by the use of $\mathrm{C}$-reactive protein (CRP). This meta-analysis was conducted to facilitate a conclusion regarding the values of CRP in the prediction of survival rate in patients with PCa.

Methods: Manuscripts were searched, identified, and collected from different databases; quality evaluation was performed according to the different search strategies involved. Information including the comparison between overall survival (OS) and cancer-specific survival (CSS) rate were retained from different studies in patients with evaluated or lowered CRP levels. Progression-free survival (PFS) was also recorded in relation to the log of CRP. Level of relevance was assessed using hazard ratio (HR) and $95 \%$ confidence interval (CI). Heterogenicity testing was performed by using Cochran's Q test and Higgins $\mathrm{I}^{2}$ statistics. When $\mathrm{P}$ value $<0.05$, the outcome was considered statistically significant.

Results: A total 12 of manuscripts were included and evaluated from the 1,523 initially identified studies. The results of OS and PFS were 1.57 (95\% CI: 1.34 to 1.85) and 1.50 (95\% CI: 1.25 to 1.81), respectively. For CSS, the combined HR and 95\% CI was 1.92 (95\% CI: 1.36 to 2.70), which revealed a significant correlation between increased level of CRP and CSS in PCa patients.
\end{abstract}

Conclusions: This meta-analysis revealed that CRP value could be one of the critical indicators to predict the survival rate of PCa patients.

Keywords: C-reactive protein (CRP); prognosis; prostate cancer (PCa); meta-analysis

Submitted Aug 03, 2021. Accepted for publication Oct 22, 2021.

doi: $10.21037 /$ tcr-21-2097

View this article at: https://dx.doi.org/10.21037/tcr-21-2097

\section{Introduction}

Cancer is currently one of the leading causes of human death. In economically developed countries, cancer has overtaken cardiovascular disease as the leading cause of death, and it is the second leading cause in many developing countries (1). Prostate cancer (PCa) is the most common urinary malignancy in older men (2). It is only surpassed by lung cancer and colorectal cancer as a cause of death among older men in Western countries, making it the third leading cause of cancer death in this demographic (3). 
In China in recent years, with the continuous growth of the aging population, the incidence rate of $\mathrm{PCa}$ has been on the rise, especially among obese or diabetic patients (4), which has piqued the attention of urologists. The prognosis of $\mathrm{PCa}$ patients mainly depends on early diagnosis and appropriate treatment. After active and effective treatment, the 5 -year survival rate of patients with PCa confined to the capsule can reach above $90 \%$; however, following treatment of metastatic PCa, the 5-year survival rate peaks at $30 \%$ (5). Therefore, early and timely diagnosis and reasonable treatment for PCa patients is very important. To date, prostate-specific antigen (PSA) is still the most widely used serum biomarker in clinical diagnosis of prostatic adenocarcinoma, and it has a strong predictive value for PCa. However, due to its high false positive rate, PSA is still the most widely used serum marker in clinical diagnosis. Guidelines published by the United States Preventive Services Task Force no longer advocates routine PSA screening of younger men (6). At the same time, PSA is not accurate in predicting patient prognosis, so more biological markers are needed to assist in the diagnosis and prognosis prediction of $\mathrm{PCa}(7)$.

C-reactive protein (CRP), a plasma protein in acute phase, is increased during the systemic inflammatory response and is one of the commonly used markers of inflammation. It is produced in the liver and is regulated by many proinflammatory cytokines, especially interleukin-6 (IL-6) (5). In fact, inflammation can play potentially dichotomous (both pro and antitumorigenic) roles depending on the nature and the cellular makeup of the immune response (8).

Normally, circulating CRP levels are low and difficult to detect in healthy people. However, an increase in circulating CRP concentration has been reported in many diseases (including infectious diseases, cardiovascular diseases, diabetes, inflammatory bowel disease, autoimmune diseases, and a variety of cancers) (9). Clinically, CRP is easy to detect and clinically operable, and it is a biological indicator that is helpful for the clinical diagnosis of $\mathrm{PCa}$ and prognostic judgment of $\mathrm{PCa}$ patients (10).

In recent years, many studies have reported that CRP plays a crucial role in the occurrence and development of PCa. This article reviews recent advances in the study of CRP and draws conclusions about the effectiveness of CRP in prognosis evaluation in PCa. We present the following article in accordance with the MOOSE reporting checklist (available at https://dx.doi.org/10.21037/tcr-21-2097).

\section{Methods}

This meta-analysis was performed according to the Metaanalysis of Observational Studies in Epidemiology Group Guideline (MOOSE) (11).

\section{Search strategy}

The databases of Medline and Embase were searched systematically from August 2013 to April 2021 in order to locate relevant research. Searching terms as C-reactive protein, CRP and prostate cancer, PCa were used to identify manuscripts which were eligible.

\section{Eligibility of manuscripts included}

\section{Inclusion criteria}

Manuscripts were included if they met the following criteria: CRP value was measured before treatment, possible correlations were evaluated between CRP value before treatment and overall survival (OS) and study design was prospective or retrospective.

\section{Exclusion criteria}

The exclusion criteria were as follows: meeting abstracts, meeting notes, reviews, letters, news reports, and laboratory studies. Studies which did not include necessary information such as $95 \%$ confidence interval (CI) and hazard ratio (HR); incomplete or duplicate studies were also excluded.

\section{Literature screening and quality evaluation}

The screening process of manuscripts and final crosschecking were completed by 2 reviewers independently. All disagreements were resolved by the agreement of both reviewers or via consultation with a third party. In order to prevent overestimation in the results, only the additional participants included in a repeated article were selected.

All manuscripts underwent quality evaluation according to the MOOSE checklist (11). The content of the checklist included: (I) precise description of included population and country; (II) accurate definition of the study design; (III) comprehensive definition of outcome evaluation, OS rate, cancer-specific survival (CSS) rate, and progressionfree survival (PFS) rate; (IV) clear definition of cut-off for CRP or accurate definition of log of CRP; (V) sufficiently duration of follow-up. These standards were used to exclude unqualified studies in order to improve the quality of this 


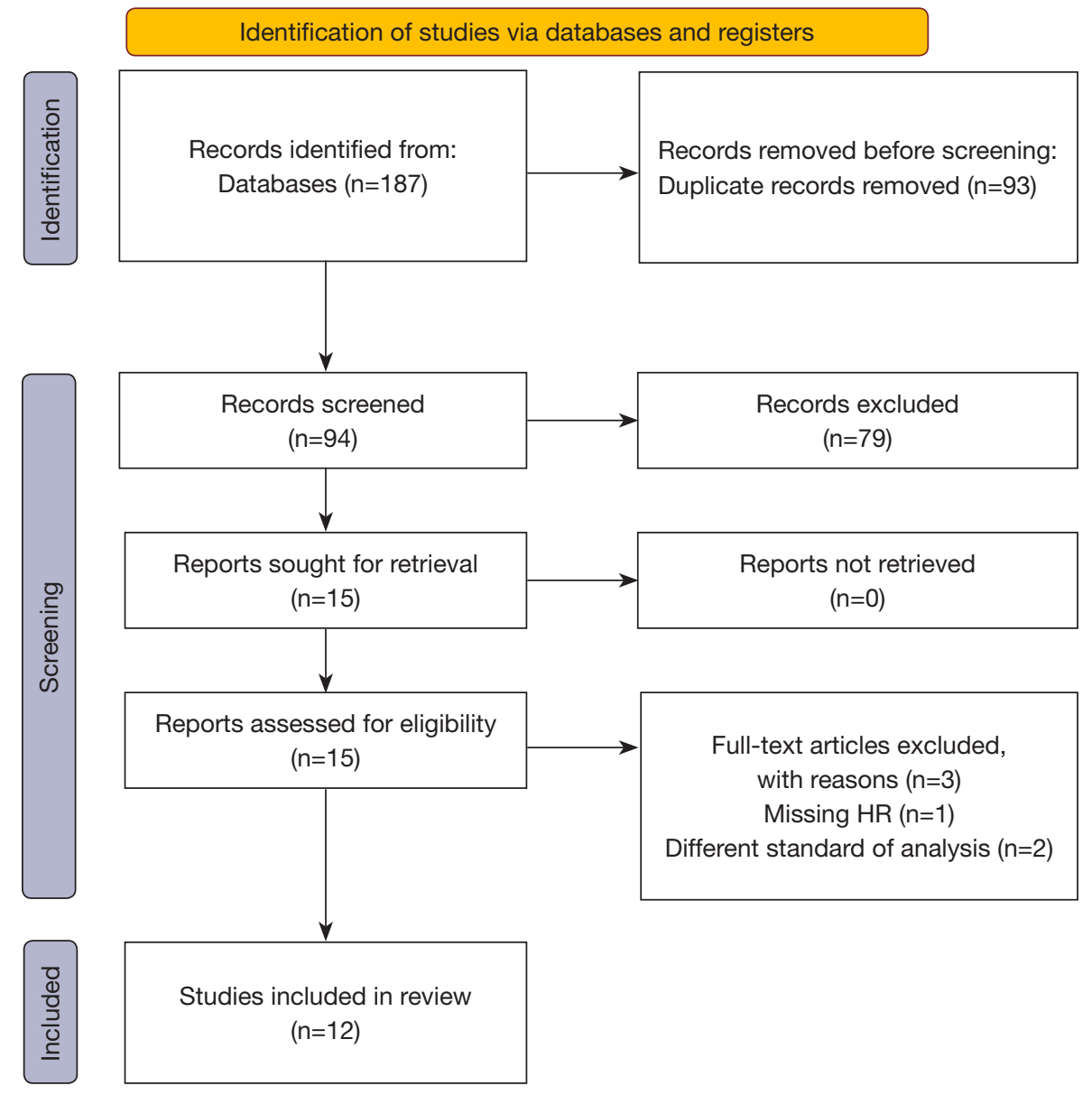

Figure 1 Flow diagram of screening process of manuscripts. HR, hazard ratio.

manuscript. The screening process is shown in Figure 1.

\section{Data extraction}

After screening of manuscripts, the following data was extracted: (I) first author, publication year, population of the study, and which country this study was held; (II) study design; (III) characteristics including staging, number of samples, and age; (IV) HR of CRP level to evaluate, OS rate, CSS rate, $\mathrm{PFS}$ rate, and their $95 \%$ CI. If data was not clearly shown, we manually extracted the total number of patient deaths against each group's sample size in order to calculate the HR (12).

\section{Statistical analysis}

Heterogenicity testing was performed by using Cochran's $\mathrm{Q}$ test and Higgins $\mathrm{I}^{2}$ statistics. When $\mathrm{P}$ value $<0.05$, the outcome was considered statistically significant. Sensitivity evaluation was performed in order to evaluate the causes of heterogenicity. Review Manager 5.3 (RevMan, Copenhagen: The Nordic Cochrane Center, The Cochrane Collaboration, 2014) was the main software used to analyze all the data and statistics.

\section{Results}

\section{Data retrieval}

After primary screening of the PubMed and Embase databases, 94 results with recorded CRP level were retrieved. After reading titles and abstracts, 79 articles were excluded due to being letters, newspapers, having important data missing, and analysis irrelevant to the topics. Some additional manuscripts were excluded due to inappropriate reporting standards; others were excluded due to too 


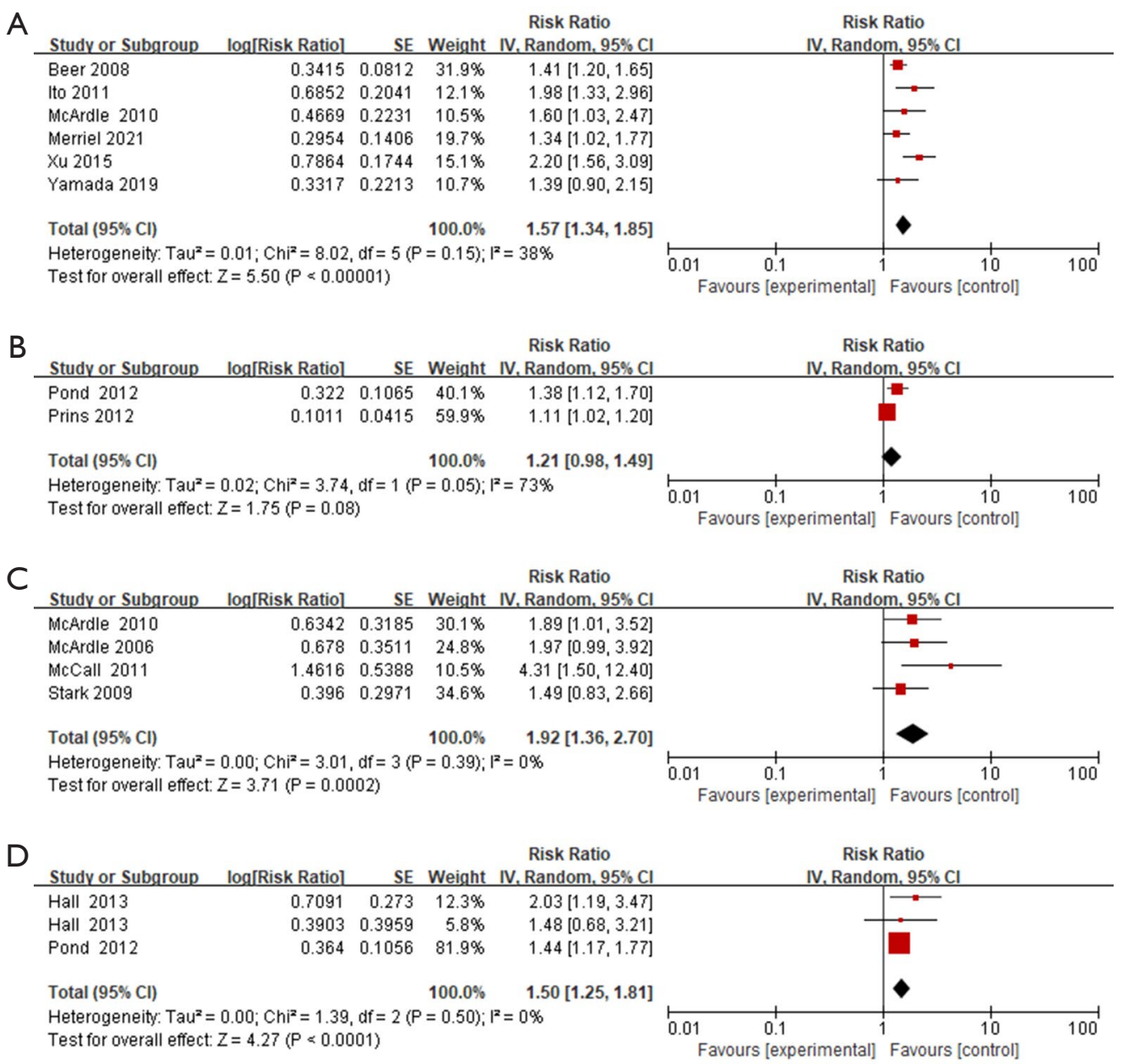

Figure 2 Forest plots of pooled outcomes in: (A) and (B) OS rate; (C) CSS rate; (D) PFS rate with HR and 95\% confidence interval in the evaluation of high and low level of serum CRP. OS, overall survival; CSS, cancer-specific survival; PFS, progression-free survival; HR, hazard ratio; CI, confidence interval; CRP, C-reactive protein.

short duration of follow-up to observe the survival rate; 2 manuscripts were excluded due to their recording of CRP-albumin ratio (CAR) and IL-6 instead of CRP level. Eventually, 12 studies were included in the meta-analysis (13-24).

\section{OS rate}

Among the studies which evaluated OS rate, there was no obvious evidence of significant heterogeneity in categorized CRP $(\mathrm{P}=0.15)$. According to the results displayed in Figure 2, the serum CRP level was relevant to the OS rate in PCa patients with combined HR estimated at about 1.57 (95\% CI: 1.34 to 1.85). However, certain evidence showed significant heterogeneity in $\log \mathrm{CRP}(\mathrm{P}=0.05)$ but not statistically significant in HR and 95\% CI 1.21 (95\% CI: 0.98 to 1.49$)$.

\section{CCS rate and PFS rate}

The random effects model was used to evaluate CSS and PFS outcomes. As shown in Figure 2, the P value in CSS and PFS was 0.39 and 0.50 , respectively, which showed insignificant heterogeneity in either CSS or PFS. For CSS, the combined level of HR and 95\% CI was calculated as 1.92 (95\% CI: 1.36 to 2.70 ), which revealed a significant correlation between increased level of CRP with PCa patients in CSS The pooled HR and 95\% CI in PFS was calculated as 1.50 (95\% CI: 1.25 to 1.81 ), which also showed a relationship between increased CRP level and PFS in PCa patients. 

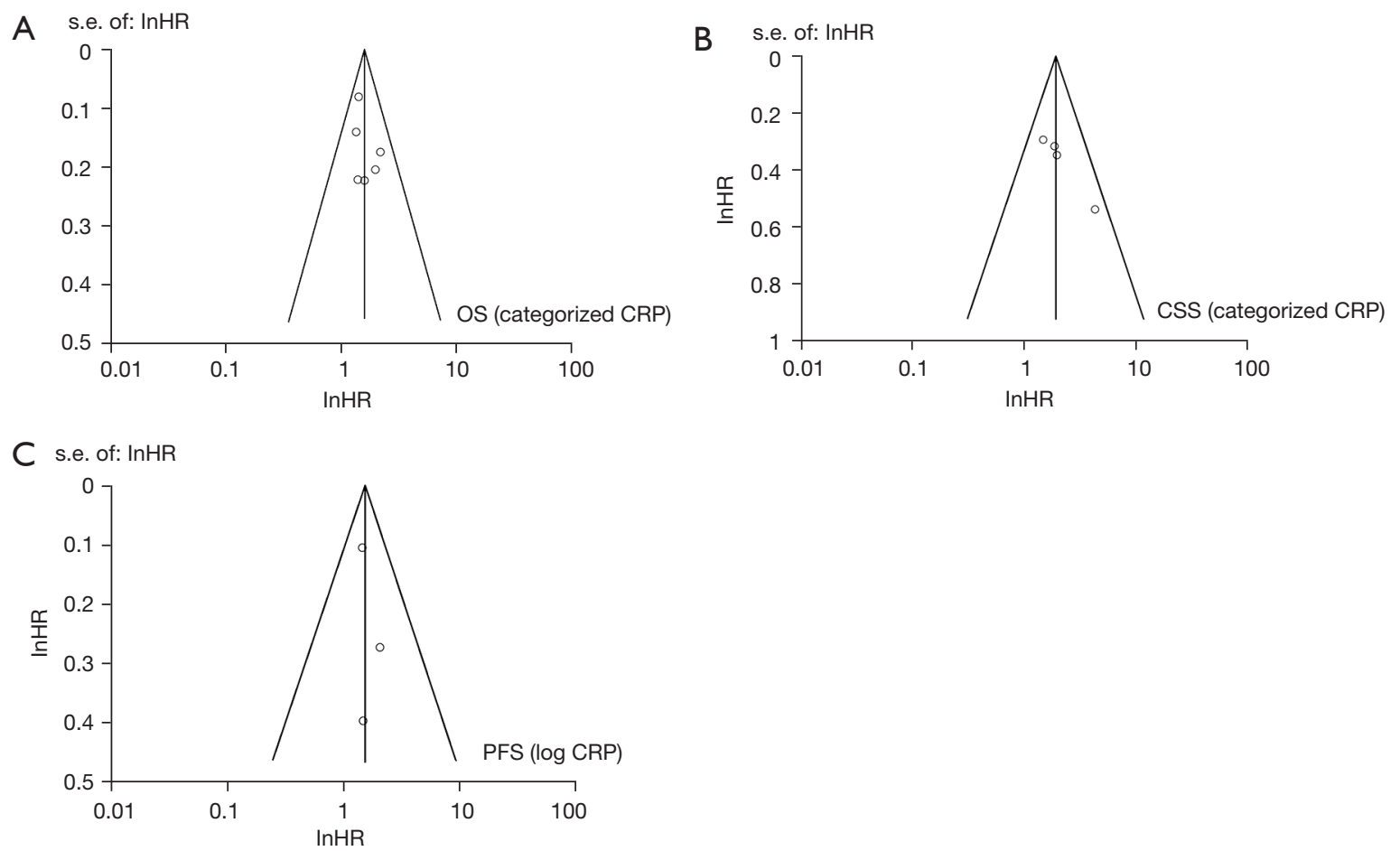

Figure 3 Funnel plots of pooled outcomes in: (A) OS rate; (B) CSS rate; (C) PFS rate. OS, overall survival; CSS, cancer-specific survival; PFS, progression-free survival; HR, hazard ratio; SE, standard error.

All the above data indicated that CRP serum level was useful and suggestive in the prediction of prognosis in $\mathrm{PCa}$ patients as one of the tumor markers.

\section{Publication bias}

To evaluate the publication bias, funnel plots with the Egger's bias indicator test were used to evaluate the included studies (25). As shown in Figure 3, two of these funnel plots were symmetrical. In conclusion, there were negative findings in the presence of significant publication bias in categorized OS rate and PFS rate.

\section{Limitations}

Most of the data on the prognosis of PCa have been produced via retrospective analysis, and few prospective and randomized controlled studies have been conducted, so circulating CRP has not yet been recognized as an independent indicator for prognosis prediction (26). However, the predictive role of circulating CRP level in prognosis and post-treatment outcome of PCa patients has been confirmed by many studies. The combination of commonly used bedside indicators and patient's circulating CRP level would be more accurate in predicting the prognosis.

\section{Discussion}

The molecular mechanism of inflammation and tumorigenesis has been a hot topic in recent years. An acute inflammatory response can protect damaged tissues, while a chronic inflammatory response may be one of the causes of tumorigenesis. The possible mechanisms include: regulating the production of free radicals to cause irreversible cell damage; accelerating cell growth by promoting DNA and cell repair; the inflammatory response creates a protective microenvironment that is also conducive to the growth of tumor cells. There have been many studies on the relationship between inflammatory response and the occurrence and development of PCa. The inflammatory marker CRP is an acute chronological serum protein synthesized by liver cells and its content amplified in inflammatory disease. Its role is to promote the movement of white blood cells and improve the activity of phagocytes to activate the complement system. Some studies have 
shown that elevated CRP levels are a risk factor for poor prognosis in patients with steroid-refractory PCa.

In addition, although several studies have evaluated the effect of serum CRP level as a prognostic factor in PCa, the results obtained have remained contradictory, and no studies have revealed the relationship between baseline CRP level and OS in Chinese patients with metastatic PCa. Previous study indicated that elevated CRP expression is significantly associated with worse PCa survival, and CRP is a strong predictor for all three survival outcomes, especially for CSS (27).

Currently, multiple studies have shown that circulating CRP levels contribute to the prediction of prognosis in many malignancies, including $\mathrm{PCa}$, and some studies have suggested that elevated cyclic CRP levels are an independent predictor of poorer prognosis in $\mathrm{PCa}$ patients $(13,23,28,29)$. Circulating CRP level is considered a strong predictor of 3 survival indicators (PFS, CSS, and OS) in patients with anterior adenocarcinoma, especially for CSS of PCa patients, circulating CRP level has a strong predictive effect (30). Graff et al. (31) summarized and analyzed previous related studies and determined the critical value of circulating CRP level in prognosis judgment of PCa. They asserted that $12 \mathrm{mg} / \mathrm{L}$ was the best critical value for prognosis judgment, and this critical value was closely correlated with patient survival rate. Patients with high circulating CRP levels had a statistically significant (83\%) increased risk of death. Xu et al. (23) believed that circulating CRP level $\geq 10 \mathrm{mg} / \mathrm{L}$ could more effectively represent a significant increase in mortality and a significant decrease in survival rate. Nakashima et al. (32) also found through multivariate analysis that circulating CRP level and bone scan results are important prognostic indicators for patients with metastatic PCa, and can be used as a standard to divide patients into high- and low-risk groups. The 5 -year survival rates for low-risk and high-risk patients were $74 \%$ and $24 \%$, respectively, which were significantly different. Taken together, the association between CRP and $\mathrm{PCa}$ is a potential but modifiable risk factor. Interventions that interfere with CRP and even inflammatory pathways may reduce the risk of $\mathrm{PCa}$ and improve prognosis and survival of PCa patients. It was shown that aspirin reduced circulating CRP levels and inflammatory reaction to drug use, and transfer of PCa and PCa mortality in patients with a negative correlation, but the clinical benefits of using chemical methods to intervene CRP and the inflammatory response in high-risk adenocarcinoma patients requires further study (27).

\section{Conclusions}

Inflammation has been repeatedly shown to be related to the occurrence and development of a variety of tumors, and CRP is a sensitive and easily measured factor in the inflammatory response. It may also influence the occurrence and development of various tumors through the related inflammatory mechanisms. In addition, due to the interaction between CRP and PCa tissue, CRP has also been considered an important mediator in the development and progression of PCa. However, circulating CRP levels are easily affected by factors such as age and smoking. At the same time, the mechanism of action between CRP and PCa has not been fully elucidated. Whether CRPdominated inflammatory factors lead to the generation and development of PCa or the stimulation of PCa to surrounding tissues leads to the increase of CRP reactivity has also been controversial. In addition, preocular studies are often limited to small, retrospective studies, and the relationship between $\mathrm{CRP}$ and $\mathrm{PCa}$ in large sample data and prospective studies has remained controversial.

Therefore, to date, circulating CRP level has not been used in the diagnosis and prediction of $\mathrm{PCa}$ alone, and further exploration of the relationship between CRP and $\mathrm{PCa}$ and its related mechanisms is required. This metaanalysis integrated the related more recent studies on the relationship between CRP and PCa. Many studies pointed out that CRP is closely related to the occurrence and development of $\mathrm{PCa}$, which is expected to be used as a new indicator for the diagnosis and prognosis of $\mathrm{PCa}$, and should be observed or trialled in clinical practice. It can also be combined with other common indicators to try to improve their overall sensitivity and specificity. At the same time, in future research on CRP and PCa, more attention should be paid to the basic research on its mechanism, so as to clarify the specific relationship and mechanism between $\mathrm{CRP}$ and $\mathrm{PCa}$, and even other malignant tumors.

\section{Acknowledgments}

Funding: Promote the hospital high-quality development fund of People's Hospital of Guang'an City, Guang'an City, Sichuan Province (21FZ013).

\section{Footnote}

Reporting Checklist: The authors have completed the MOOSE reporting checklist. Available at https://dx.doi. 
org/10.21037/tcr-21-2097

Conflicts of Interest: All authors have completed the ICMJE uniform disclosure form (available at https://dx.doi. org/10.21037/tcr-21-2097). The authors have no conflicts of interest to declare.

Ethical Statement: The authors are accountable for all aspects of the work in ensuring that questions related to the accuracy or integrity of any part of the work are appropriately investigated and resolved.

Open Access Statement: This is an Open Access article distributed in accordance with the Creative Commons Attribution-NonCommercial-NoDerivs 4.0 International License (CC BY-NC-ND 4.0), which permits the noncommercial replication and distribution of the article with the strict proviso that no changes or edits are made and the original work is properly cited (including links to both the formal publication through the relevant DOI and the license). See: https://creativecommons.org/licenses/by-nc-nd/4.0/.

\section{References}

1. Bray F, Ferlay J, Soerjomataram I, et al. Global cancer statistics 2018: GLOBOCAN estimates of incidence and mortality worldwide for 36 cancers in 185 countries. CA Cancer J Clin 2018;68:394-424.

2. Sung H, Ferlay J, Siegel RL, et al. Global Cancer Statistics 2020: GLOBOCAN Estimates of Incidence and Mortality Worldwide for 36 Cancers in 185 Countries. CA Cancer J Clin 2021;71:209-49.

3. Thurner EM, Krenn-Pilko S, Langsenlehner U, et al. The elevated C-reactive protein level is associated with poor prognosis in prostate cancer patients treated with radiotherapy. Eur J Cancer 2015;51:610-9.

4. Xie DD, Chen $\mathrm{YH}, \mathrm{Xu} \mathrm{S}$, et al. Low vitamin D status is associated with inflammation in patients with prostate cancer. Oncotarget 2017;8:22076-85.

5. Elsberger B, Lankston L, McMillan DC, et al. Presence of tumoural C-reactive protein correlates with progressive prostate cancer. Prostate Cancer Prostatic Dis 2011;14:122-8.

6. US Preventive Services Task Force; Grossman DC, Curry SJ, et al. Screening for Prostate Cancer: US Preventive Services Task Force Recommendation Statement. JAMA 2018;319:1901-13.

7. Hayashi T, Fujita K, Tanigawa G, et al. Serum monocyte fraction of white blood cells is increased in patients with high Gleason score prostate cancer. Oncotarget 2017;8:35255-61.

8. Sfanos KS, Hempel HA, De Marzo AM. The role of inflammation in prostate cancer. Adv Exp Med Biol 2014;816:153-81.

9. Lee S, Choe JW, Kim HK, et al. High-sensitivity C-reactive protein and cancer. J Epidemiol 2011;21:161-8.

10. Stikbakke E, Richardsen E, Knutsen T, et al. Inflammatory serum markers and risk and severity of prostate cancer: The PROCA-life study. Int J Cancer 2020;147:84-92.

11. Stroup DF, Berlin JA, Morton SC, et al. Meta-analysis of observational studies in epidemiology: a proposal for reporting. Meta-analysis Of Observational Studies in Epidemiology (MOOSE) group. JAMA 2000;283:2008-12.

12. Parmar MK, Torri V, Stewart L. Extracting summary statistics to perform meta-analyses of the published literature for survival endpoints. Stat Med 1998;17:2815-34.

13. Beer TM, Lalani AS, Lee S, et al. C-reactive protein as a prognostic marker for men with androgen-independent prostate cancer: results from the ASCENT trial. Cancer 2008;112:2377-83.

14. Prins RC, Rademacher BL, Mongoue-Tchokote S, et al. $\mathrm{C}$-reactive protein as an adverse prognostic marker for men with castration-resistant prostate cancer (CRPC): confirmatory results. Urol Oncol 2012;30:33-7.

15. Ito M, Saito K, Yasuda Y, et al. Prognostic impact of C-reactive protein for determining overall survival of patients with castration-resistant prostate cancer treated with docetaxel. Urology 2011;78:1131-5.

16. McArdle PA, Mir K, Almushatat AS, et al. Systemic inflammatory response, prostate-specific antigen and survival in patients with metastatic prostate cancer. Urol Int 2006;77:127-9.

17. Stark JR, Li H, Kraft P, et al. Circulating prediagnostic interleukin-6 and C-reactive protein and prostate cancer incidence and mortality. Int J Cancer 2009;124:2683-9.

18. McArdle PA, Qayyum T, McMillan DC. Systemic inflammatory response and survival in patients with localised prostate cancer: 10-year follow-up. Urol Int 2010;85:482.

19. McCall P, Catlow J, McArdle PA, et al. Tumoral C-reactive protein and nuclear factor kappa-B expression are associated with clinical outcome in patients with prostate cancer. Cancer Biomark 2011-2012;10:91-9.

20. Pond GR, Armstrong AJ, Wood BA, et al. Ability of C-reactive protein to complement multiple prognostic 
classifiers in men with metastatic castration resistant prostate cancer receiving docetaxel-based chemotherapy. BJU Int 2012;110:E461-8.

21. Hall WA, Nickleach DC, Master VA, et al. The association between C-reactive protein (CRP) level and biochemical failure-free survival in patients after radiation therapy for nonmetastatic adenocarcinoma of the prostate. Cancer 2013;119:3272-9.

22. Merriel SWD, Ingle SM, May MT, et al. Retrospective cohort study evaluating clinical, biochemical and pharmacological prognostic factors for prostate cancer progression using primary care data. BMJ Open 2021;11:e044420.

23. Xu L, Zhao Q, Huang S, et al. Serum C-reactive protein acted as a prognostic biomarker for overall survival in metastatic prostate cancer patients. Tumour Biol 2015;36:669-73.

24. Yamada Y, Sakamoto S, Rii J, et al. Prognostic value of an inflammatory index for patients with metastatic castrationresistant prostate cancer. Prostate 2020;80:559-69.

25. Egger M, Davey Smith G, Schneider M, et al. Bias in meta-analysis detected by a simple, graphical test. BMJ 1997;315:629-34.

26. Sciarra A, Gentilucci A, Salciccia S, et al. Prognostic value of inflammation in prostate cancer progression and

Cite this article as: Du J, Lan J, Xiong J, Yang H, Xu X, Tang C, Huang G, Ying Q, Mu J, Hu Q. Efficiency of C-reactive protein in prognosis evaluation of prostate cancer: a systematic review and meta-analysis. Transl Cancer Res 2021;10(10): 4432-4439. doi: 10.21037/tcr-21-2097 response to therapeutic: a critical review. J Inflamm (Lond) 2016;13:35.

27. Liu Y, Chen JQ, Xie L, et al. Effect of aspirin and other non-steroidal anti-inflammatory drugs on prostate cancer incidence and mortality: a systematic review and metaanalysis. BMC Med 2014;12:55.

28. Dai J, Tang K, Xiao W, et al. Prognostic significance of C-reactive protein in urological cancers: a systematic review and meta-analysis. Asian Pac J Cancer Prev 2014;15:3369-75.

29. Liao SG, Cheng HH, Lei Y. C-Reactive Protein is a Prognostic Marker for Patients with Castration-Resistant Prostate Cancer. Oncol Res Treat 2016;39:266-71.

30. Liu ZQ, Chu L, Fang JM, et al. Prognostic role of C-reactive protein in prostate cancer: a systematic review and meta-analysis. Asian J Androl 2014;16:467-71.

31. Graff JN, Beer TM, Liu B, et al. Pooled Analysis of C-Reactive Protein Levels and Mortality in Prostate Cancer Patients. Clin Genitourin Cancer 2015;13:e217-21.

32. Nakashima J, Kikuchi E, Miyajima A, et al. Simple stratification of survival using bone scan and serum C-reactive protein in prostate cancer patients with metastases. Urol Int 2008;80:129-33.

(English Language Editor: J. Jones) 